\title{
Cryogenic scintillators in searches for extremely rare events
}

\author{
V B Mikhailik ${ }^{1}$ and H Kraus \\ Department of Physics, University of Oxford, Keble Road, Oxford OX1 3RH, UK \\ E-mail: vmikhai@hotmail.com
}

Received 12 October 2005, in final form 17 January 2006

Published 3 March 2006

Online at stacks.iop.org/JPhysD/39/1181

\begin{abstract}
Inorganic scintillators are important elements of a new type of cryogenic phonon scintillation detector (CPSD) being developed for single particle detection. These detectors, exhibiting superior energy resolution and the ability to identify the type of interaction in an event, are considered to be the next generation of instrumentation in the search for extremely rare events. This paper presents the latest results of our research on cryogenic scintillators for CPSD applications in the search for dark matter. The paper gives a description of the concept of direct dark matter detection and the operation principles of CPSD, discusses the major material requirements and summarizes the results of investigations over a wide temperature range of the luminescence and scintillation properties of tungstates $\left(\mathrm{CaWO}_{4}\right.$ and $\left.\mathrm{ZnWO}_{4}\right)$, molybdates $\left(\mathrm{CaMoO}_{4}, \mathrm{MgMoO}_{4}\right.$ and $\left.\mathrm{CdMoO}_{4}\right)$ and Ti-doped $\mathrm{Al}_{2} \mathrm{O}_{3}$.
\end{abstract}

\section{Introduction}

Searches for extremely rare events, such as non-baryonic dark matter, $0-\mathrm{v}$ double-beta decay and radioactive decay of very long-living isotopes, are high up on the agenda of the particle physics community. These 'needles in a haystack' could give an insight into the existence of new fundamental particles, new physics and how the universe is built. Advancing this research requires detection techniques capable of distinguishing weak and extremely rare interaction signals over significant radioactivity from natural backgrounds. Research and development are underway for the implementation of new instrumentation in low-background particle physics experiments.

Recent progress in the field of low-temperature detection techniques has led to the development of highly sensitive energy resolving cryogenic detectors capable of detecting the energy deposited in their absorber by particle or radiation interaction. It is the very high resolving power of cryogenic detectors that makes them interesting for an increasing number of applications in fundamental [1-3] and applied sciences $[4,5]$.

Further progress in the development of cryogenic detectors for rare event searches relies on the implementation

\footnotetext{
1 Author to whom any correspondence should be addressed.
}

of event type discrimination for detection of radiation and particles. One of the most promising techniques is the event-by-event discrimination through measurement of the phonon and scintillation responses in cryogenic detectors. This technique provides the unique advantage of background identification and discrimination that is crucially important for rare-event and low-background physics experiments. In combination with the other advantages of cryogenic phonon detectors, such as greatly enhanced energy resolution and low threshold, this feature is a major benefit. Consequently, the next generation of 'hybrid' cryogenic phonon-scintillation detectors (CPSD) is being successfully used by the CRESST and ROSEBUD Collaborations in the search for dark matter $[6,7]$ and studies of very long-lived isotopes $[8,9]$. The CUORE Collaboration is considering the implementation of the CPSD technique in the search for double beta decay [10]. European underground rare event calorimeter array (EURECA) is a massive cryogenic dark matter search experiment planned to start construction around 2008/09, in which groups across Europe join forces and aim to concentrate on a single detection technique as far as cryogenic detectors are concerned. Given the advantages of CPSDs, there is a good chance for this technique to prevail.

Scintillating crystals are a key element in CPSDs, and the identification and characterization of potential scintillation materials is an important objective for future experiments 
in this field. Although there has always been interest in scintillators, operating them at low temperatures is an entirely new aspect. This is a new area of research which has not been mainstream in scintillator studies hitherto.

Our group at the University of Oxford has a longstanding tradition of working in the field of low-temperature detectors for experimental particle astrophysics, and a couple of years ago we have initiated a new research program into cryogenic scintillators for particle physics and medicine. In this paper we present an overview of our investigations aimed at finding, characterizing and optimizing inorganic scintillators for cryogenic application. As our primary motivation is the development of scintillation materials for CPSDs used in dark matter search, we first discuss the principles of direct detection of dark matter, detector concept and the material requirements imposed by the former. We then summarize the results of studies of luminescence and scintillation properties over the temperature range 9-300 K for several prospective materials: $\mathrm{CaWO}_{4}$ (as reference), $\mathrm{ZnWO}_{4}$, $\mathrm{CaMoO}_{4}, \mathrm{MgMoO}_{4}, \mathrm{CdMoO}_{4}$ and Ti-doped aluminium oxide. It needs to be noted that the results of practical applications of cryogenic scintillators showed that such studies as well as scintillation parameters measured at $9 \mathrm{~K}$ can be used for the assessment of scintillator performance at millikelvin temperatures. In this temperature interval the major scintillation characteristics (light yield and decay time) follow the general trends, which are governed by the processes that start at much higher temperatures. Therefore the indicative values of these parameters can be obtained by extrapolation of the data obtained at $\sim 10 \mathrm{~K}$. This is also confirmed by published results on light yield and decay time constant in the millikelvin range [10-12].

\section{Concept of direct WIMP detection}

There is evidence that the total mass of the Universe is significantly higher than the one observed directly (see review by [13] and reference therein). The most immediate evidence results from measurements of the radial distribution of the rotation speeds of spiral galaxies. These results indicate that a large amount of non-luminous (dark) matter is necessary to explain the flat rotation curves. The main candidates for dark matter favoured by modern cosmological and supersymmetric theories are weakly interacting massive particles (WIMPs) [14]. Albeit very rare, WIMPs interact with ordinary matter via the elastic scattering process. Therefore direct WIMP detection can be accomplished by measurements of the recoil energy they impart to nuclei.

When assuming spin-independent coherent scattering, the cross section of the interaction is proportional to the square of the mass number $\left(A^{2}\right)$ of the nucleus. Theoretical estimates of spin-independent interaction cross sections of WIMP give values of $\leqslant 10^{-7}$ pbarn $[15,16]$ which effectively means less than a few events per kilogram of detector mass per month. Another type of interaction is spin-dependent scattering which can be detected using materials with nuclei exhibiting non-zero spin $(J)$. Detectors with the capability of using different target materials, thereby exploiting the dependence of cross sections on $A$ and $J$, are of primary importance for the confirmation of a WIMP signal. It needs to be stressed that it is the capability of
CPSDs to utilize different materials that makes the technology especially useful for dark matter search experiment.

A further feature of WIMP detection originates from the necessity to measure a very low recoil energy (of the order of a few tens of kilo-electron-volts or less) that imposes stringent requirements on the detector's threshold. An additional complication in low-background experiments is the background from natural radioactivity and cosmic rays dominating in this energy region which gives an event rate far greater than the signal rate. Much effort is therefore required to shield the detector well and to select scintillator materials which themselves exhibit low background. In summary it is obvious that direct WIMP detection is a challenging goal and that it requires truly advanced detection technology.

\section{Operation principles of the CPSD technique for WIMP search}

Currently there are more than a dozen experiments trying to detect WIMPs using different experimental approaches. Passive background suppression is achieved with a low background installation and a deep underground location. A consensus has been reached that dark matter searches need to have some kind of active background rejection capability to achieve competitive results. In this regard, the CPSD technique offering the possibility of unrivalled sensitivity and discrimination between electron and nuclei recoils is of particular interest.

Conceptually, the CPSD technique consists of simultaneous measuring of the phonon signal and scintillation light produced in a target in interaction events [17]. The discrimination between nuclear and electron recoils is possible because the fraction of energy converted into scintillation light in the course of electron recoils (caused by background) is larger compared with the nuclear recoils (typical of WIMP interaction). The actual ratio of the light signals produced depends on the recoiling nuclei.

CPSDs can be viewed as a combination of two cryogenic detectors cooled to millikelvin temperature. One of them is a massive block of target material, i.e. scintillating crystal, and the other is a cryogenic detector optimized for the detection of absorbed scintillation photons (see figure 1(a). Both detectors are read out by superconducting phase transition thermometers (SPT) operating as calorimeters [4]. Energy deposition in crystal (massive scintillating absorber or light detector) causes a temperature rise of the electron system in the SPT. The SPT consists of a thin superconducting film that is stabilized at a temperature within its transition from the super to normal state. Figure 1 $(b)$ demonstrates how a small variation in temperature of the SPT's electron system translates into a noticeable change of resistance, which can be measured with a superconducting quantum interference device (SQUID) readout circuit.

The energy resolution of the detector based on SPT temperature sensors is roughly proportional to temperature; lowering of the transition temperature leads to an enhancement of the detector sensitivity [18]. So far the lowest operating temperature is obtained with tungsten SPTs exhibiting a superconducting transition in the region of $10-15 \mathrm{mK}$. This low operating temperature allowed energy resolutions of 1 and $2 \mathrm{keV}$ (FWHM) for the phonon and light detector, respectively, 


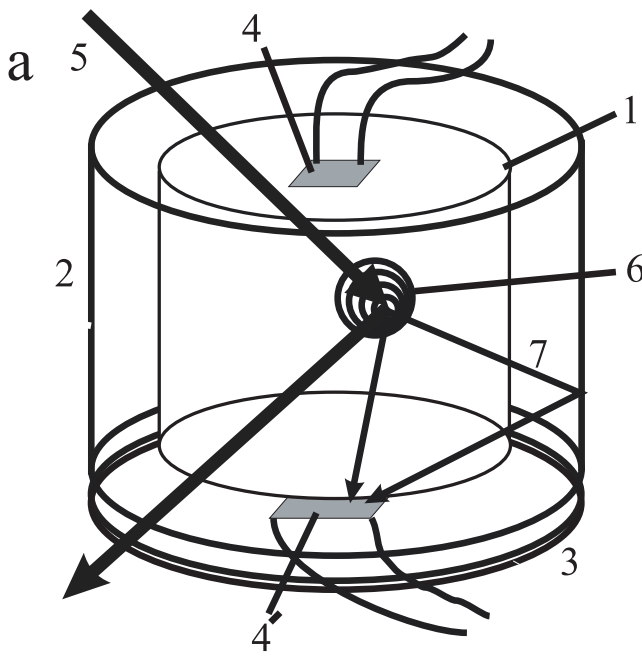

b

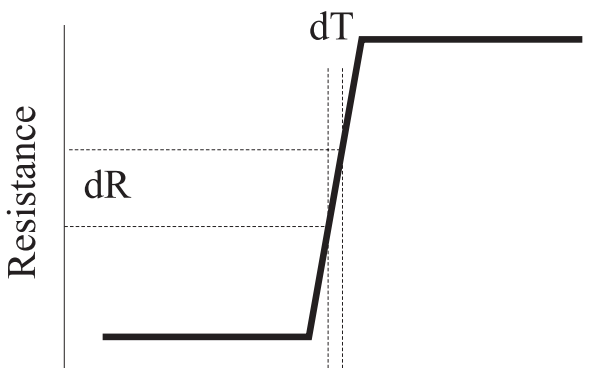

Temperature, $\mathrm{mK}$

Figure 1. (a) Schematic of a cryogenic detector used for simultaneous measurement of scintillation and phonons from a scintillating crystal. 1-scintillating crystal, 2-reflector, 3-light detector, 4, 4'-SPT-film, 5-particle, 6-phonons and 7-light. (b) Schematic of SPT. Biased in the middle of its transition, a small change in temperature translates into a substantial change of resistance.

in the CRESST setup with $\mathrm{CaWO}_{4}$ scintillators [6]. This translates into an event discrimination threshold of $12 \mathrm{keV}$. One can appreciate the importance of transition temperature when comparing with the latest results of another dark matter experiment (ROSEBUD) that operates a similar setup at $60 \mathrm{mK}$. An energy resolution of $38 \mathrm{keV}$ (FWHM) and a discrimination threshold of $74 \mathrm{keV}$ have been reported for their $\mathrm{CaWO}_{4}$ detector [7].

\section{Material requirements}

As is emphasized above, the important advantage of the CPSD technique is the possibility of using different target materials in one common setup, thereby exploiting the material signature of WIMP events. Because of this there is a need for dedicated research aiming to develop cryogenic scintillating detectors capable of meeting stringent requirements regarding low intrinsic radioactivity and having the capability to measure very small energy depositions.

When facing such a task it is very helpful to establish a set of acceptance criteria which allows the scope of the search to be narrowed by eliminating less suitable materials. As concerns the cryogenic scintillators, a high scintillation yield in the millikelvin temperature range is a key criterion. Nonetheless, other requirements, such as radio purity, surface properties and thermodynamic properties of the lattice, can be equally important. The acceptance criteria for the cryogenic scintillators thought to be a possibility in low-background experiments can be summarized as follows.

(1) High scintillation yield in the millikelvin temperature range. The higher the scintillation yield the lower is the energy threshold for which discrimination between electron and nuclear recoil is possible for a given confidence level. Lower energy threshold translates into less exposure (mass $\times$ time) needed to reach certain sensitivity levels of cross sections for WIMP-nucleon scattering.

(2) Radio purity. The contamination of radioactive nuclei in the scintillators must be kept as low as possible. This brings about a stringent limitation on the composition of the material. For instance, rare-earths elements, alkali metals, lead, cadmium, bismuth and thallium containing traces of natural radioactive isotopes must be eliminated.

(3) Surface properties. The crystal surface must be nonhygroscopic to reduce external contamination. It has to be resistant to high-temperature vacuum treatment due to the nature of the thin film deposition technique.

(4) Thermodynamic properties of the lattice. Because of the necessity to cool the cryogenic detector to millikelvin temperatures the specific heat of the lattice has to be low. This precludes the use of paramagnetic ions.

(5) Cost. Given the perspectives of future dark matter experiments having a target mass of $100 \mathrm{~kg}$ and more, the cost of the material cannot be excessive.

A survey of the performance of existing commercial scintillators in view of these requirements gives the following.

Doped halide scintillators (NaI-Tl, CsI-Tl, CsI-Na, $\mathrm{CaF}_{2}-\mathrm{Eu}$ ) do not satisfy either conditions (2) or (3) or both. Moreover, the light yield of impurity luminescence often decreases with temperature deteriorating hereby the merit of these materials. This makes these scintillators unsuitable for use in the cryogenic experiment.

The light yield of pure halide scintillators $\left(\mathrm{BaF}_{2}, \mathrm{CsI}\right)$ is constant at low temperatures and could be considered reasonably high. However they have the same problems as the group of scintillators above, associated with insufficient radio purity and surface stability.

Ce-doped scintillators (YAP, LSO, GSO, YAG, $\mathrm{LaCl}_{3}$, etc) are totally unacceptable because of the very high intrinsic radioactive background inherent in the rare-earth host matrix and the dopant.

With regard to these problems in particular our interest focused on self-activated scintillators. These materials exhibit a high light yield at low temperatures; they are stable and affordable. However care must be taken with regard to the radio purity of particular compounds. For example, the radio purity of BGO, CWO and PWO is inferior because, as mentioned above, natural $\mathrm{Bi}, \mathrm{Cd}$ and $\mathrm{Pb}$ inevitably contain detrimental radioactive isotopes, i.e. members of the radioactive decay of the U-Th chains. 


\section{Luminescence and scintillation characterization of materials for CPSDs}

What becomes immediately clear after such an analysis is that there are only few conventional scintillators left which can be considered for CPSD application in the search for rare events. $\mathrm{CaWO}_{4}$ is currently the favourite in dark matter searches: it exhibits sufficiently high light yield at low temperatures, it is stable, free of natural radioactive isotopes and can be produced with satisfactory purity. Interest is now turning to other members of the tungstate family as well as molybdates. It is thought that the members of these groups could satisfy the principal requirements of suitable mechanical and surface properties, high light yield and appropriate isotopic content implying low intrinsic radioactivity and hence might be attractive materials for CPSD. Another interesting material belongs to the class of doped crystals, here $\mathrm{Al}_{2} \mathrm{O}_{3}-\mathrm{Ti}$. The comprehensive characterization of the luminescence and scintillation properties of these materials should provide the necessary information for a further assessment of their potential for this application. In the following section we will bring together the results of such studies.

\subsection{Experimental}

In our experiments we examined samples grown by the Czochralski technique from raw materials having purities of $99.99 \%$ or better (tungstates) and $99.95 \%$ (molybdates), respectively. The Ti-doped $\mathrm{Al}_{2} \mathrm{O}_{3}$ researched by us is a commercial sample of scintillation quality. All the samples for measurements were of size $5 \times 5 \times 1 \mathrm{~mm}^{3}$; they were polished to optical quality. For luminescence characterization of the crystals under different excitation conditions the following experimental facilities were used.

(1) Monochromator with a $\mathrm{D}_{2}$-lamp and higher-harmonic YAG laser excitation source (for experimental details see [19, 20]).

(2) SUPERLUMI station at HASYLAB (Hamburg, Germany) operating in the energy range $4-40 \mathrm{eV}$ [21].

(3) Mobile luminescence end-station (MoLES) at SRS Daresbury laboratory [22] that uses monochromatic X-ray photons provided by beamlines MPW6.1 (150-400 eV) and $4.2(2-4 \mathrm{keV})$.

(4) Spectroscopic setup comprising an x-ray tube with tungsten anticathode operating at $35 \mathrm{keV}$ and providing bremsstrahlung x-ray radiation [23].

(5) Experimental setup for the measurements of scintillator characteristics (light yield and decay time constant) using multiple photon counting coincidence technique [24].

The luminescence, reflection, excitation spectra, light yield and decay time characteristics of crystals were measured as functions of temperature in the temperature range 9-300 K. All luminescence spectra presented are corrected for dispersion $\left(\lambda^{2}\right.$-correction) and instrumental response of the detection systems.

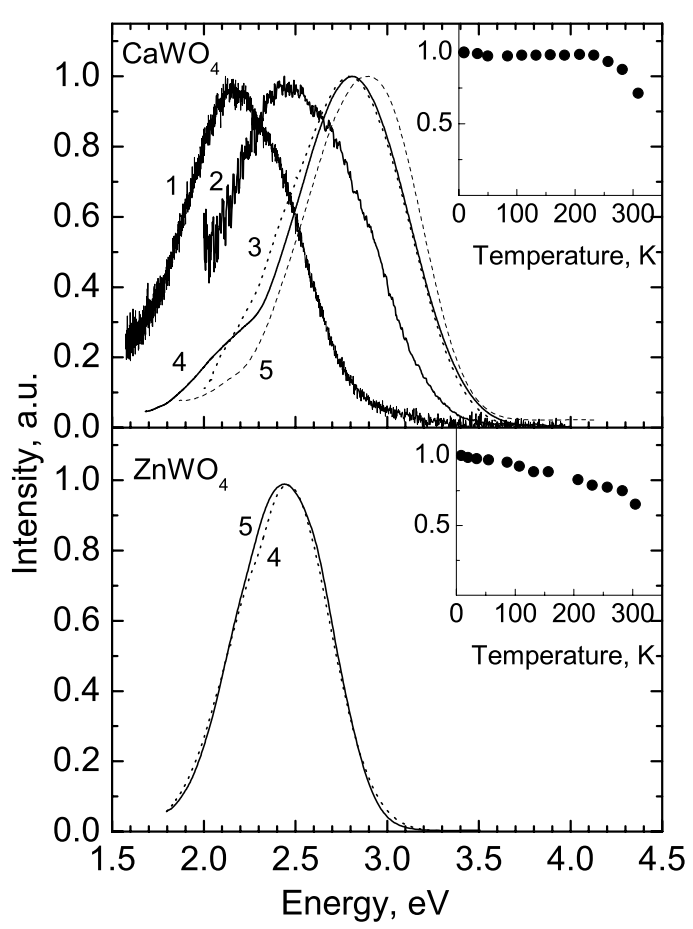

Figure 2. Luminescence spectra of $\mathrm{CaWO}_{4}$ and $\mathrm{ZnWO}_{4}$ measured for different excitations: $1-4.4 \mathrm{eV}, 2-4.5 \mathrm{eV}, 3-7.1 \mathrm{eV}, 4-31 \mathrm{eV}$ and $5-3.2 \mathrm{keV}(T=9 \mathrm{~K})$. Insets show the change of the emission intensity as a function of temperature measured for excitation with $3.2 \mathrm{keV} \mathrm{x}$-ray photons.

\subsection{Tungstates $\left(\mathrm{CaWO}_{4}\right.$ and $\left.\mathrm{ZnWO}_{4}\right)$}

Tungstate and molybdate crystals have a long history of practical application as phosphors, laser hosts and scintillators [25-30]. In such applications the luminescence properties of the materials are of particular importance, and this resulted in a large number of previous research works. Therefore the luminescence properties of the crystals under interest have been investigated most extensively. The earlier studies have been done mainly using ultraviolet, $\mathrm{x}$-ray or particle excitation $[25,26,30-37]$. The results of investigations using timeresolved techniques under excitation with VUV synchrotron radiation [38-40], pulsed UV [41,42] and x-ray sources $[43,44]$ have been published recently. Although the emission characteristics of the materials have been examined in quite a few studies down to LHeT, this has been driven exclusively by academic interest. On the other side motivation of our research is more pragmatic: it implies investigation of the material properties in view of their possible application at the low temperatures. Therefore the emphasis is laid on the assessment of the low temperature characteristics of the material in view of their possible application in CPSD technique.

As $\mathrm{CaWO}_{4}$ has been selected in the course of previous studies and is used presently as a CPSD in searches for dark matter, we carried out a thorough examination of this compound. This included luminescence studies using different excitations, i.e. two-photon, ultra violet $[19,20]$, vacuum ultraviolet and x-ray [45], scintillation characterization [24] as well as theoretical studies of the lattice dynamics [46].

Figure 2 shows the emission spectra of $\mathrm{CaWO}_{4}$ measured at a temperature of $9 \mathrm{~K}$ under different excitation conditions. 
At high energy the x-ray excitation $\left(E_{\mathrm{ex}}>10^{3} \mathrm{eV}\right)$ spectrum shows structureless broad band emission centred at $2.9 \mathrm{eV}$ with a pronounced extension towards lower energies. This band is due to the electronic transitions of the charge transfer type within the $\left(\mathrm{WO}_{4}\right)^{2-}$ anion complex and is interpreted as an emission of self-trapped molecular excitons [30,33,34].

In the tungstate crystals with sheelite structure optical transitions occur within the oxyanion molecular complex $(\mathrm{WO})_{4}^{2-}\left(\mathrm{T}_{\mathrm{d}}\right.$ symmetry) between the ${ }^{1} \mathrm{~A}_{1}$ ground state and the singlet $\left({ }^{1} \mathrm{~T}_{1},{ }^{1} \mathrm{~T}_{2}\right)$ and triplet $\left({ }^{3} \mathrm{~T}_{1},{ }^{3} \mathrm{~T}_{2}\right)$ levels. The lower-lying excited electron configuration associated with the metal d-orbital contains four states, specifically ${ }^{1} \mathrm{~T}_{2}>{ }^{1} \mathrm{~T}_{1}>{ }^{3} \mathrm{~T}_{2} \geqslant{ }^{3} \mathrm{~T}_{1}$ in order of decreasing energy. It is generally accepted that electric dipole allowed transitions $\left({ }^{1} \mathrm{~A}_{1} \rightarrow{ }^{1} \mathrm{~T}_{2},{ }^{1} \mathrm{~T}_{1}\right)$ account for the fundamental absorption edge of $\mathrm{CaWO}_{4}$ while the lower-lying triplets ${ }^{3} \mathrm{~T}_{2}$ and ${ }^{3} \mathrm{~T}_{1}$ are responsible for the radiative transitions [33,34].

With increasing temperature the emission maxima shift towards higher energy and the luminescence spectra exhibit broadening; such thermal changes are mostly due to interaction of the $\left(\mathrm{WO}_{4}\right)^{2-}$ emission centre with lattice vibrations [19]. It has been found that the luminescence spectra of $\mathrm{CaWO}_{4}$ show a noticeable change with the excitation condition. As the excitation energy of the radiation decreases the luminescence spectra exhibit a shift towards low energy and an additional structure overlapping the low-energy part of the main emission band can be identified (see figure 2). When excited below the band gap energy of $\mathrm{CaWO}_{4}$, which is currently assigned to $5.2 \mathrm{eV}$ [19], the low-energy green emission band dominates. It is argued that this emission of calcium tungstate is due to a structural defect of the lattice, an oxygen-deficient complex, $\mathrm{WO}_{3}[34,41,47]$. Given the penetration depth of the radiation progressively increasing with the energy of photons impinging on the samples, it is suggested that these defects are mainly localized in a thin $(\sim 100 \mathrm{~nm})$ surface layer of the $\mathrm{CaWO}_{4}$ crystal [45]. This allowed us to conclude that under high-energy excitation, when the crystal is used as a scintillator, the intrinsic (blue) emission band controls the major characteristics of $\mathrm{CaWO}_{4}$ as well as the temperature dependence of the light yield (see inset in figure 2). The latter shows very little variation over the temperature range 9 to $250 \mathrm{~K}$; above that, thermal quenching causes a decrease of the light output.

Zinc tungstate was the second compound that attracted much of our attention. This material has suitable composition in regard to radio purity issues, and given the fact that it is a commercial scintillator, it was thought it was well worthy of examination. $\mathrm{ZnWO}_{4}$ has wolframite structure and the luminescence properties of this crystal are noticeably different from $\mathrm{CaWO}_{4}$. At $9 \mathrm{~K}$ the luminescence spectrum spans the range 1.8 to $3.0 \mathrm{eV}$ showing a prominent emission peak at $2.45 \mathrm{eV}$ (figure 2)). This emission is associated with the radiative transitions between tungsten and oxygen within the $\left(\mathrm{WO}_{6}\right)^{6-}$ molecular complex [37]. Analysis of the experimental results demonstrates that across the excitation conditions used, the luminescence spectra of $\mathrm{ZnWO}_{4}$ crystal are very similar. The same holds for the temperature dependences of the luminescence light yield; they exhibit very similar trends irrespective of the excitation energy (see inset in figure $2(b) . \mathrm{ZnWO}_{4}$ has a maximum light output at

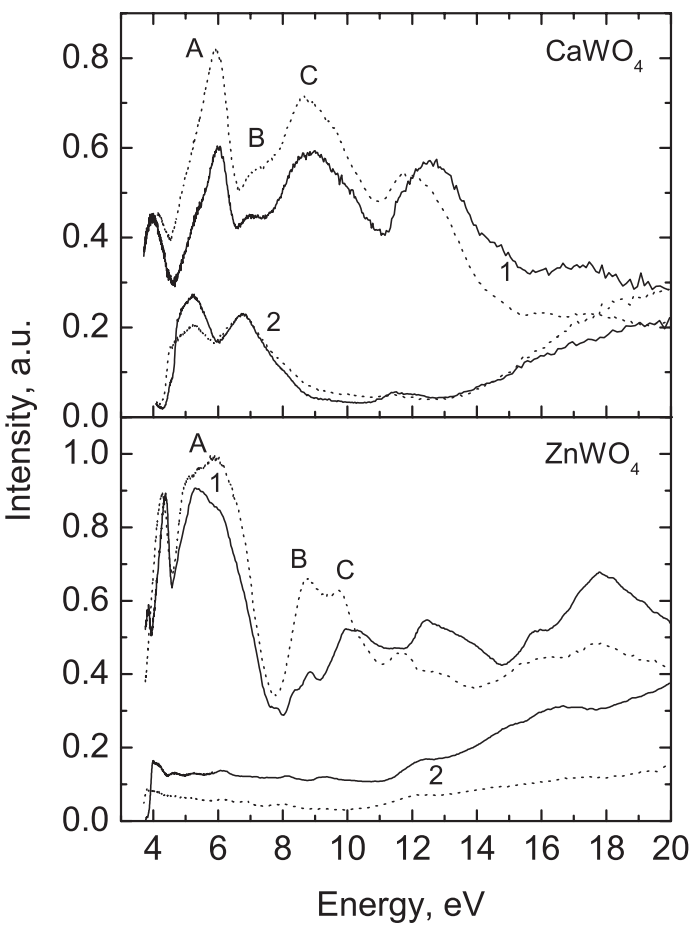

Figure 3. Reflection (1) and luminescence excitation spectra (2) of $\mathrm{CaWO}_{4}$ and $\mathrm{ZnWO}_{4}$ crystals. Solid line $-T=9 \mathrm{~K}$ and dotted line- $T=295 \mathrm{~K}$.

a temperature of $9 \mathrm{~K}$; it decreases gradually with increasing temperature. At a temperature of $9 \mathrm{~K}$ and $3.2 \mathrm{keV}$ excitation energy, the relative light output of $\mathrm{ZnWO}_{4}$ is assessed to be $\sim 10 \%$ higher than that of $\mathrm{CaWO}_{4}$. At room temperature the light yield of both samples is virtually identical. The same measurements of the light yield as a function of temperature were carried out at $320 \mathrm{eV}$ excitation energy. At this energy, the ratio for the light yield $(\sim 115 \%)$ obtained for the two crystals at $9 \mathrm{~K}$ is the same within the experimental accuracy $( \pm 10 \%)$. These results show that the intrinsic light yield of $\mathrm{ZnWO}_{4}$ at low temperature is higher than that of $\mathrm{CaWO}_{4}$.

Figure 3 shows the reflection and excitation spectra of $\mathrm{CaWO}_{4}$ and $\mathrm{ZnWO}_{4}$ crystals measured at $9 \mathrm{~K}$. In the low energy part of the reflection spectra there is a recognizable spurious structure, namely a trough at $4.5 \mathrm{eV}$, which is due to reflection from the rear surface of the sample and from the sample holder right behind. Recent calculations of band structure of tungstate crystals $[48,49]$ provide a theoretical background for the identification of the reflection spectra features. For both crystals the crystal splitting of the W 5d levels by tetrahedral $\left(\mathrm{CaWO}_{4}\right)$ and octahedral crystal fields $\left(\mathrm{ZnWO}_{4}\right)$ yields a group of $e$ and $t_{2}$ states. The relative position of these levels is inverted in both crystals. In sheelite $\left(\mathrm{CaWO}_{4}\right)$ the lower part of the conduction band is associated with the well-separated $e$ states while the upper peak is due to $t_{2}$ states located $\sim 1.5 \mathrm{eV}$ above. This produces the band (A) at $5.9 \mathrm{eV}$ and a shoulder (B) at $\sim 7 \mathrm{eV}$ in the reflectivity spectra of $\mathrm{CaWO}_{4}$ that can be assigned to the transition from the $\mathrm{O} 2 \mathrm{p}$ valence band to the empty W $5 \mathrm{~d}$ states. The $4 \mathrm{~d}$ Ca states start to contribute to the total density-of-states $2-3 \mathrm{eV}$ above and they dominate the upper part of the conduction band. Therefore the 
characteristic peak (C) at $8.7 \mathrm{eV}$ is assigned to the transition from the valence band terminating at the $4 \mathrm{~d}$ calcium levels.

Due to the difference in the conduction band structure the reflection spectrum of wolframite $\left(\mathrm{ZnWO}_{4}\right)$ and its interpretation is appreciably different. The first reflection band (A) at $5.8 \mathrm{eV}$ is attributed to the transitions from the valence band to the $t_{2}$ states of the $\mathrm{W} 5 \mathrm{~d}$ band. This band is broad: it spans a range of more than $2 \mathrm{eV}$, being consistent with the calculated results of [49]. In line with this interpretation two bands (B) and (C) arising at 8.7 and $9.7 \mathrm{eV}$ represent the $e$ states which are split into two levels.

The luminescence excitation spectra of $\mathrm{CaWO}_{4}$ and $\mathrm{ZnWO}_{4}$ exhibit features that are consistent with the other optical properties of these crystals. The sheelite $\mathrm{CaWO}_{4}$ crystal gives rise to two overlapping emission bands (intrinsic and defect) and can also be identified in the excitation spectrum. The defect (green) emission is mainly excited in the narrow near-edge region (4.3-4.7 eV) while intrinsic emission dominates at higher energies [19]. Since both emissions strongly overlap, the excitation spectrum of $\mathrm{CaWO}_{4}$ measured at $2.9 \mathrm{eV}$ represents a superposition of two excitation spectra that is clearly noticeable in the edge region. No such feature is observed in the excitation spectrum of $\mathrm{ZnWO}_{4}$; it starts steeply at the energies that correspond to the absorption edge of the crystal $(\sim 3.8 \mathrm{eV})$. This agrees with the concept of a sole emission centre in this crystal [45]. In the region $5-8 \mathrm{eV}$ in $\mathrm{CaWO}_{4}$ corresponding to the charge-transfer transition within the oxyanion molecular complex the correlated electron-hole pairs are efficiently created and recombine. The rise in the excitation efficiency seen above $13 \mathrm{eV}$ in $\mathrm{CaWO}_{4}(11 \mathrm{eV}$ in $\mathrm{ZnWO}_{4}$ ) is associated with the beginning of the multiplication of the electronic excitation [50].

The decay time kinetics of $\mathrm{CaWO}_{4}$ has been measured using both optical and particle ( ${ }^{241} \mathrm{Am}$ source) excitation. Only one decay time constant $\tau=8.1 \pm 0.5 \mu$ s was found at room temperature under optical excitation [19]. Figure 4(a) shows the scintillation decay time spectra of $\mathrm{CaWO}_{4}$ and $\mathrm{ZnWO}_{4}$ measured at 295 and $9 \mathrm{~K}$ using an ${ }^{241} \mathrm{Am}$ source. A sum of two exponentials was used to fit the experimental data for $\mathrm{CaWO}_{4}$ although in the temperature range $20-50 \mathrm{~K}$ it was necessary to add a third component to achieve the best fit. Figure $4(b)$ shows the temperature dependence of the decay components. At room temperature the decay time constants of $\mathrm{CaWO}_{4}$ are $\tau_{1}=1.4 \pm 0.1 \mu \mathrm{s}$ and $\tau_{2}=8.9 \pm 0.2 \mu \mathrm{s}$, respectively. Figure 4(b) shows that the decay curves evolve gradually in the temperature range $350-50 \mathrm{~K}$ as the temperature decreases. At $T<50 \mathrm{~K}$ the character of the decay changes drastically: a long component starts to emerge and it becomes very pronounced below $20 \mathrm{~K}$, the decay time constant being $390 \pm 20 \mu \mathrm{s}$ at $9 \mathrm{~K}$. This behaviour is characteristic of the decay time spectra of $\mathrm{CaWO}_{4}$ which is caused by the existence of a metastable level a few milli-electron-volts below the emitting level [36]. At low temperature the thermal depopulation of this level is suppressed causing the probability of radiative recombination to decrease significantly, which slows down the whole emission process. It needs to be noted that the scintillation decay time constant of $\mathrm{CaWO}_{4}$ at millikelvin temperatures is found to be ca. $400 \pm 100 \mu$ s [11], which is in agreement with our findings.

The best fit to the decay time curves of $\mathrm{ZnWO}_{4}$ was achieved using three exponentials, and at room temperature
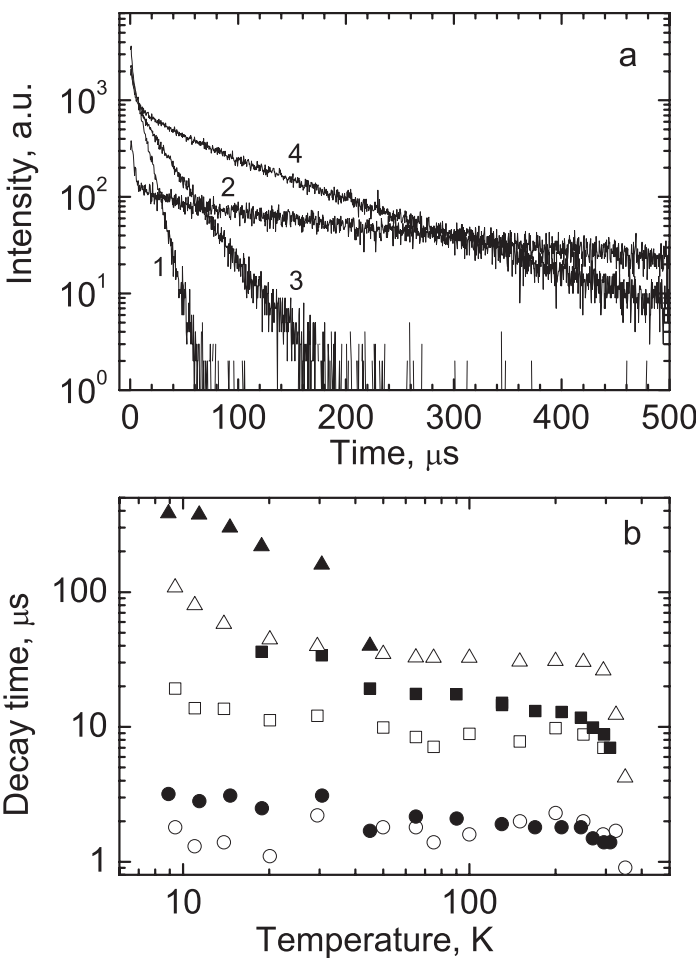

Figure 4. (a) Decay time spectra of scintillation in $\mathrm{CaWO}_{4}(1,2)$ and $\mathrm{ZnWO}_{4}(3,4)$ measured at temperature $295 \mathrm{~K}(1,3)$ and $9 \mathrm{~K}$ $(2,4)$. (b) Temperature dependence of the decay time constants in $\mathrm{CaWO}_{4}$ (filled symbols) and $\mathrm{ZnWO}_{4}$ (open symbols)

the decay time constants are equal to $\tau_{1}=1.3 \pm 0.1 \mu \mathrm{s}$, $\tau_{2}=5.6 \pm 0.3 \mu \mathrm{s}$ and $\tau_{3}=25.7 \pm 0.3 \mu \mathrm{s}$, respectively. There is also another marked difference in the decay time constant behaviour of $\mathrm{ZnWO}_{4}$ at low temperature when comparing with $\mathrm{CaWO}_{4}$. The decay time spectrum becomes longer but evolves rather gradually and does not exhibit very rapid change. Thus the long decay time constant of $\mathrm{ZnWO}_{4}$ at $9 \mathrm{~K}$ is shorter than that of $\mathrm{CaWO}_{4}$, being $110 \pm 15 \mu \mathrm{s}$; even at millikelvin temperatures the decay time is reported as being $\sim 200 \mu$ s [42], still shorter than that of $\mathrm{CaWO}_{4}$. This finding indicates that the timing characteristic of zinc tungstate at low temperature is much better than that of $\mathrm{CaWO}_{4}$.

Summarizing the results of experimental studies of these two scintillators the conclusion was drawn that $\mathrm{ZnWO}_{4}$ exhibits an improvement of scintillation efficiency and better timing characteristics when cooled to low temperatures. Overall, $\mathrm{ZnWO}_{4}$ appears to be an excellent complement to $\mathrm{CaWO}_{4}$ in cryogenic dark matter searches based on the detection of phonon and scintillation signals. The combination of these two tungstates could form the basis for the first multi-target detector capable of WIMP identification through a material signature [51].

\subsection{Molybdates $\left(\mathrm{MgMoO}_{4}, \mathrm{CaMoO}_{4}\right.$ and $\left.\mathrm{CdMoO}_{4}\right)$}

As already stated, molybdates represent the second group of materials that are interesting for CPSD technology. Due to the high luminescence light yield, appropriate isotopic content and suitable mechanical and surface properties, some of the representatives of this group may offer a suitable choice in experimental searches for rare events. Of particular interest is 


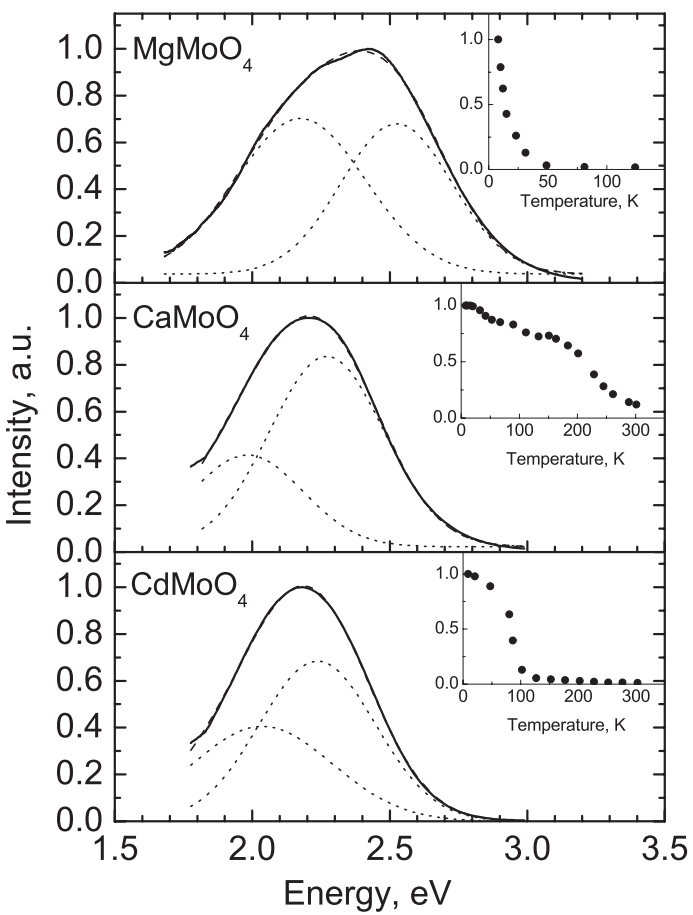

Figure 5. Luminescence spectra of $\mathrm{MgMoO}_{4}, \mathrm{CaMoO}_{4}$ and $\mathrm{CdMoO}_{4}$ measured at $9 \mathrm{~K}$ and excitation with $3.2 \mathrm{keV}$ x-ray photons. The dotted lines show two-Gaussian decompositions of the emission spectra. Insets show the temperature change of the emission intensity.

$\mathrm{CaMoO}_{4}$ which is homological to $\mathrm{CaWO}_{4}$ and is therefore an indispensable complementary compound for exploiting material signature, hereby extending the sensitivity of dark matter experiments. Another interest in molybdates arises from their suitability for experiments searching for double-beta decay; ${ }^{100} \mathrm{Mo}$ is considered one of the most promising isotopes for the detection of this phenomenon [2]. The respective experiment that uses scintillating $\mathrm{CaMoO}_{4}$ has been launched recently [52].

$\mathrm{CaMoO}_{4}$ and $\mathrm{CdMoO}_{4}$ have sheelite-type structure being identical to $\mathrm{CaWO}_{4}$ in this respect. Single crystals of $\mathrm{MgMoO}_{4}$ have monoclinic symmetry. However it needs to be emphasized that in this structure each Mo is surrounded by four $\mathrm{O}$ sites in approximately tetrahedral coordination. Due to this common feature - the existence of the isolated $\mathrm{MO}_{4}$ tetrahedra-the electronic structure of all molybdates under investigation is very similar, permitting comparison of their optical properties. The applicability of this approach has been confirmed by theoretical calculations of the band structure of $\mathrm{MgMoO}_{4}$ [53], $\mathrm{CaMoO}_{4}$ [48] and $\mathrm{CdMoO}_{4}$ [49].

Figure 5 shows luminescence spectra of the crystals measured using monochromatic VUV excitation $(31 \mathrm{eV})$. At low temperatures, molybdate crystals exhibit broad luminescence bands with a maximum at $2.4\left(\mathrm{MgMoO}_{4}\right), 2.2$ $\left(\mathrm{CaMoO}_{4}\right)$ and $2.15 \mathrm{eV}\left(\mathrm{CdMoO}_{4}\right)$. Of these crystals only $\mathrm{CaMoO}_{4}$ has a noticeable light yield at room temperature. Starting at low temperature, $\mathrm{CaMoO}_{4}$ shows almost constant light yield up to temperature of $100 \mathrm{~K}$ which then gradually decreases (see inset in figure 2). The quenching temperature $T_{\mathrm{q}}$ (temperature when the emission intensity exhibits half of its initial value) is found to be $210 \mathrm{~K}$. In $\mathrm{MgMoO}_{4}$

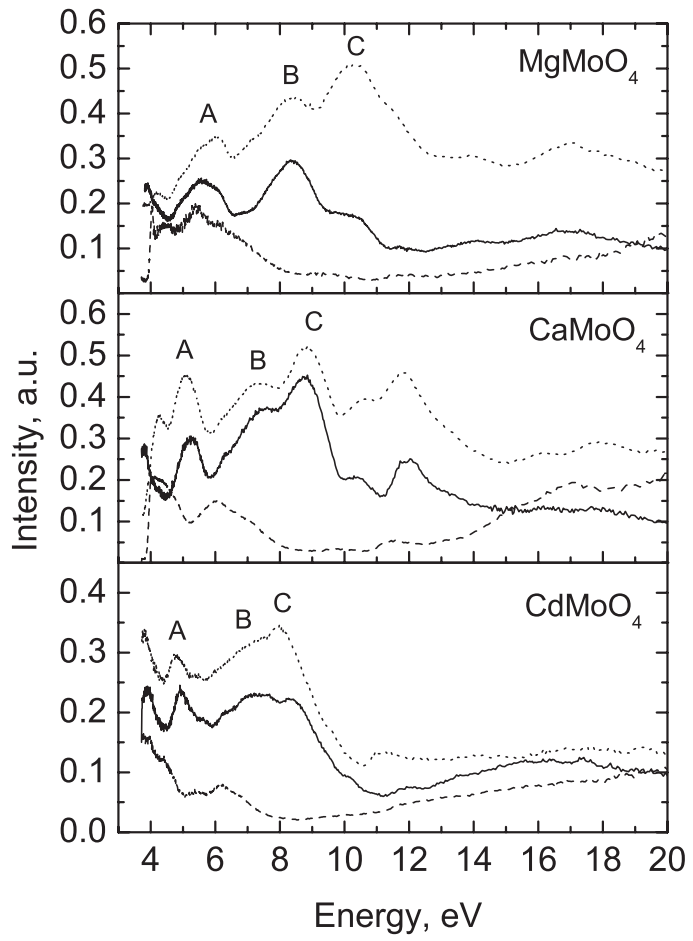

Figure 6. Reflection ( - and $\cdots \cdot \cdot)$ ) and luminescence excitation spectra (- - - ) of $\mathrm{MgMoO}_{4}, \mathrm{CaMoO}_{4}$ and $\mathrm{CdMoO}_{4}$ crystals. Solid and dashed lines $-T=9 \mathrm{~K}$, dotted line $-T=295 \mathrm{~K}$.

thermal quenching manifests itself at a very low temperature $\left(T_{\mathrm{q}}=20 \mathrm{~K}\right) . \mathrm{CdMoO}_{4}$ exhibits an intermediate quenching temperature, namely $T_{\mathrm{q}}=120 \mathrm{~K}$.

The emission spectra of the crystals under test have a composite character, and an adequate fit to the experimental results is obtained using two Gaussians (see figure 5). Given the similarity of the crystal structure of molybdates and $\mathrm{CaWO}_{4}$ as well as the analogous tetrahedral configuration of the oxyanion molecular complex, it is commonly accepted that the emission characteristics of the molybdate crystals can be interpreted in terms of the same model. Therefore the dominating opinion is that the high-energy band is associated with the radiative recombination of the electron-hole pairs localized at the $\mathrm{MoO}_{4}^{2-}$ group [32]. Consequently the lowenergy part of the spectrum is tentatively assigned to defect emission. In addition there are also considerations indicating that Jahn-Teller interaction might have an effect on the structure of the lowest emitting states of the crystals with scheelite structure [35,54]. This effect makes up the emission spectrum composite of several emission bands. Very recently this prediction has been confirmed through experimental studies of time-resolved luminescence spectra of $\mathrm{PbWO}_{4}$ [55]. It was shown that the main luminescence band consists of four emission bands with different decay times. The longlived (millisecond) low-energy band is the emission of defect centres, while the three other resolved sub-bands constituting the blue emission represent the transitions from different adiabatic potential energy surfaces (APES). Therefore it is very possible that this effect can manifest itself in molybdates.

Figure 6 presents the reflection spectra of the crystals. At low energies, each reflection spectrum exhibits a prominent peak (A) at around 5.5, 5.1 and $4.9 \mathrm{eV}(T=9 \mathrm{~K})$ which shifts 
to higher energies in order of decreasing radius of the alkalineearth cation $\left(\mathrm{MgMoO}_{4}, \mathrm{CaMoO}_{4}\right.$ and $\mathrm{CdMoO}_{4}$, respectively). The next band (B) is located approximately $2 \mathrm{eV}$ higher, i.e. at $8.4 \mathrm{eV}, 7.4 \mathrm{eV}$ and $7.1 \mathrm{eV}$ and an intense reflection band (C) emerges at $10.2 \mathrm{eV}, 8.8 \mathrm{eV}$ and $8.3 \mathrm{eV}$, respectively. The effect of cation shift is much more pronounced for the latter band. The bands in the high-energy part of the reflection spectrum $(>12 \mathrm{eV})$ are poorly resolved and weak.

The features of the reflection spectra of the molybdate crystals can be interpreted on the basis of current knowledge of their electronic structure $[48,49,53]$. The top of the valence band of molybdates is dominated by $2 p$-states of oxygen while the lowest unoccupied states are composed of the Mo 4d-states split in two sets of bands with $e$ and $t$ symmetry. The lower peak in the density-of-the-states is associated with $e$ states, while the upper one represents $t$ states. These bands have localized character with energy separation between the maxima of about $2 \mathrm{eV}$. Therefore, the two low-energy bands $\mathrm{A}$ and $\mathrm{B}$ in the reflection spectra of molybdates can be assigned to the electronic transitions from the valence $\mathrm{O} 2 \mathrm{p}$-states to the Mo $4 \mathrm{~d}$-states which have different symmetry. The transitions to the low-energy band form an interband edge, providing a rough estimate of the band gap of the material under examination. At higher energies theoretical calculations predict the contribution of alkaline cation states to the conduction band. Consequently, the intense high-energy band $\mathrm{C}$ is likely to be caused by transitions from the valence band to the highest-lying conduction band states, originating from the nd-states of alkaline-earth cation. The change of this reflection peak position for different cations is consistent with the general trend in the position of the atomic-like ndlevels of alkaline-earth cations in solids. The high-energy structure above $12 \mathrm{eV}$ can be interpreted as transitions from the valence band to the highest-lying unoccupied $5 \mathrm{~s}$ and $5 \mathrm{p}$ orbital of molybdenum [56]. The excitation of molybdates is in general most efficient in the energy range 4 to $8 \mathrm{eV}$ where exciton and correlated electron-hole pairs are created. Under excitation in this energy range the valence $\mathrm{O} 2 \mathrm{p}$ electrons are promoted to the empty $4 \mathrm{~d}$ molybdenum states in the course of charge-transfer transitions. Their subsequent recombination yields the characteristic intrinsic emission of the $\mathrm{MoO}_{4}^{2-}$ anion complex [57].

The temperature dependence of the scintillation decay kinetics of the molybdates was investigated under excitation with an ${ }^{241} \mathrm{Am}$ source. The example in figure 7(a) shows the decay time spectrum of $\mathrm{CaMoO}_{4}$ measured at 295 and $9 \mathrm{~K}$. It is found that the decay curves exhibit a complex character with a well-defined fast slope at the beginning of the luminescence pulse and a slow exponential tail. The best fit of the experimental results at $T<200 \mathrm{~K}$ can be obtained using the sum of three exponentials while two exponentials were sufficient for fitting in the region of thermal quenching. The decay time constants obtained from such a fit as a function of temperature are presented in figure 7(b).

The features of the decay kinetics of molybdate crystals can be explained taking into account the similarity of the structure with that of $\mathrm{CaWO}_{4}$. This implies the similarity of the energy structure of the emitting centre in $\mathrm{CaWO}_{4}$ and $\mathrm{CaMoO}_{4}$. Thus in the molybdate and tungstate crystals with sheelite
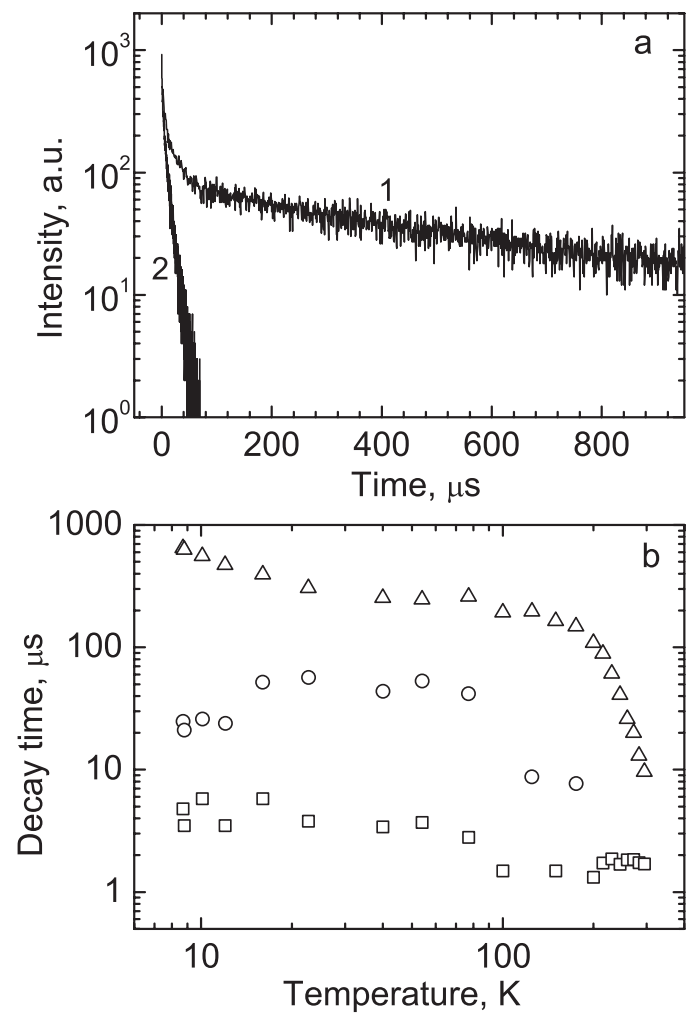

Figure 7. (a) Decay time spectra of scintillation in $\mathrm{CaMoO}_{4}$ measured at $9 \mathrm{~K}(1)$ and $295 \mathrm{~K}(2)$. (b) Temperature dependence of the two decay time constants.

structure orbitally allowed ${ }^{3} \mathrm{~T}_{1}$ and ${ }^{3} \mathrm{~T}_{2} \rightarrow{ }^{1} \mathrm{~A}_{1}$ transitions within the oxyanion molecular complex $\mathrm{MO}_{4}^{2-}(\mathrm{M}=\mathrm{W}, \mathrm{Mo})$ are responsible for the emission. It is thought that APES originating from the different triplet levels give rise to the radiative transitions with different probabilities that explain the appearance of three emission components in the decay [58]. The decrease of the decay time constants at low temperatures is consistent with the fact that the emitting state in sheelite crystals is composed of two levels of which the lowest one is metastable $[32,42]$. At low temperature this level serves as an electron trap that accounts for the decrease in the probability of the radiative decay.

Finally the assessment of the emission efficiency of molybdates at $9 \mathrm{~K}$ has been done using $\mathrm{x}$-ray $(3.2 \mathrm{keV}$ photons) and photoexcitation $(31 \mathrm{eV})$. The relative light yield of the crystal under examination is $\sim 30\left(\mathrm{MgMoO}_{4}\right), 30$ $\left(\mathrm{CaMoO}_{4}\right)$ and $80 \%\left(\mathrm{CdMoO}_{4}\right)$ with respect to $\mathrm{CaWO}_{4}$ at $31 \mathrm{eV}$ photoexcitation. It should be noted that these numbers should be considered very conservative estimates since in this study merely the experimental molybdate samples were examined in contrast to the conventional $\mathrm{ZnWO}_{4}$ and $\mathrm{CaWO}_{4}$ scintillator with a long legacy of research and development work. One might therefore expect that the light yield characteristics of the molybdates can be improved significantly by optimization of the crystal production technique. Overall these results suggest that molybdates appear to be very suitable materials for cryogenic rare event search experiments that rely on the simultaneous detection of phonons and scintillation photons. 


\subsection{Ti-doped $\mathrm{Al}_{2} \mathrm{O}_{3}$}

In our search for cryogenic scintillators capable of meeting the challenging requirements of forthcoming dark matter experiments, we turned our attention to Ti-doped aluminium oxide. Pure $\mathrm{Al}_{2} \mathrm{O}_{3}$ has already proved to be an excellent phonon detector [59] and it is thought that Ti-doped $\mathrm{Al}_{2} \mathrm{O}_{3}$, which is known as a very efficient laser material, can be adapted as CPSD.

The luminescence response of the crystals to high energy excitation (monochromatic VUV and x-ray radiation) over a wide temperature range was investigated $[60,61]$. In addition to the thoroughly studied emission band of $\mathrm{Ti}^{3+}$ in the nearIR region $(1.7 \mathrm{eV})$ and the weak blue emission at $2.9 \mathrm{eV}$, we detected a UV emission band at $4.3 \mathrm{eV}$ (see figure $8(a)$ ). This band accounts for approximately one-third of the integrated emission light yield of the crystal. The decay time spectrum of this band exhibits a single exponential decay ( $\tau=138 \mathrm{~ns}$ at
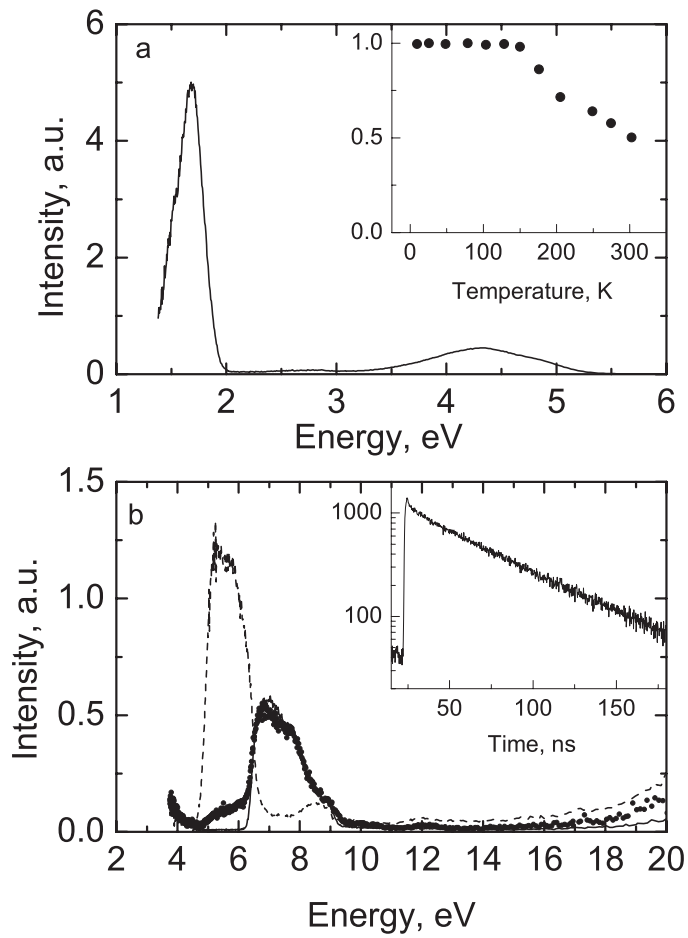

Figure 8. (a) Luminescence spectra of $\mathrm{Al}_{2} \mathrm{O}_{3}-\mathrm{Ti}$ measured under excitation with $3.2 \mathrm{keV}$ x-ray photons at $T=9 \mathrm{~K}$. The inset shows the temperature dependence of the integrated emission intensity. (b) Excitation spectra of $\mathrm{Al}_{2} \mathrm{O}_{3}$-Ti monitored for different emission bands at $T=9 \mathrm{~K}$. Dashed line, $2.9 \mathrm{eV}$; solid symbols, $1.7 \mathrm{eV}$; solid line, $4.3 \mathrm{eV}$. Inset shows decay time spectrum of the $4.3 \mathrm{eV}$ band measured at $T=300 \mathrm{~K}$.
$T=300 \mathrm{~K}$ ) with a very pronounced temperature dependence; it increases with decreasing temperature.

For the first time the excitation spectra of all emission bands were measured over the $4-20 \mathrm{eV}$ spectral range and it was clearly shown that the $2.9 \mathrm{eV}$ emission band can be efficiently excited in the spectral region from 4.6 to $\sim 7 \mathrm{eV}$ (figure $8(b)$ ). Two possible models that explain the origin of this band are considered, i.e. charge transitions $2 \mathrm{pO}^{2-} \rightarrow$ $3 \mathrm{dTi}^{4+}$ or photo-ionization of $\mathrm{Ti}^{3+}$. The proximity of the excitation threshold of these processes precludes unambiguous interpretation. On the other hand it is shown that the near-IR luminescence of $\mathrm{Ti}^{3+}$ is mainly stimulated in the region from 6.2 to $\sim 9 \mathrm{eV}$ and, importantly, the excitation spectra of the near IR and UV bands are essentially the same in this spectral range. By taking into account the spectral and decay characteristics of the UV emission, we suggested that this band is associated with radiative decay of excitons localized at $\mathrm{Ti}^{3+}$ ions.

The total light yield dependence as a function of temperature was investigated and it was found to be constant below $150 \mathrm{~K}$. It was shown that the $\mathrm{Al}_{2} \mathrm{O}_{3}-\mathrm{Ti}$ scintillator can be used as an $\mathrm{x}$-rays detector in the range $15-60 \mathrm{keV}$. The low temperature scintillation yield of $\mathrm{Al}_{2} \mathrm{O}_{3}-\mathrm{Ti}(0.20 \mathrm{wt} . \%)$ is found to be $2400 \pm 200 \mathrm{ph} \mathrm{MeV}^{-1}$ [61]. An estimate gives that the integrated luminescence light yield of the sample with the lower titanium concentration $(0.07 \mathrm{wt} . \%)$ is twice as large and hence a substantial improvement of this parameter is expected by optimizing the concentration of the luminescent impurity. This assessment is very encouraging, moving this material into the category of prospective absorbers for CPSD.

\section{Conclusion}

Low-temperature detectors are being developed for single particle detection with excellent energy resolution threshold and the ability of identification of the type of interaction event. This creates a need for materials suitable for the concept of simultaneous detection of scintillation and phonons. The search for and development of materials for CPSDs is becoming an important objective of scintillator studies.

Motivated by this we analysed the prospects of different scintillation materials for experiments searching for rare events, selected several potential candidates and summarized the results of their luminescence and scintillation characteristics over a wide temperature range (see table 1). Inspection of the results of these investigations shows that there are quite a few materials that might represent a real possibility for CPSD technology as far as rare event search experiments are concerned. Of these materials $\mathrm{CaWO}_{4}$ and $\mathrm{ZnWO}_{4}$, which are well-developed scintillators, are the most appealing

Table 1. Luminescence and scintillation characteristics of crystals at 300 and $9 \mathrm{~K}$.

\begin{tabular}{llllllll}
\hline & \multicolumn{2}{c}{ Emission peak, nm } & & \multicolumn{2}{c}{ Decay time, $\mu \mathrm{s}$} & \multicolumn{2}{c}{ Light yield $^{\mathrm{a}}$} \\
\cline { 2 - 3 } \cline { 6 - 8 } Material & $T=300 \mathrm{~K}$ & $T=9 \mathrm{~K}$ & & $T=300 \mathrm{~K}$ & $T=9 \mathrm{~K}$ & $T=300 \mathrm{~K}$ & $T=9 \mathrm{~K}$ \\
\hline $\mathrm{CaWO}_{4}$ & 420 & 420 & & 9 & 390 & 0.7 & 1.0 \\
$\mathrm{ZnWO}_{4}$ & 490 & 510 & & 25 & 110 & 0.7 & 1.1 \\
$\mathrm{CaMoO}_{4}$ & 540 & 540 & & 10 & 640 & 0.05 & 0.3 \\
$\mathrm{CdMoO}_{4}$ & - & 550 & & - & 470 & - & 0.8 \\
$\mathrm{Al}_{2} \mathrm{O}_{3}-\mathrm{Ti}$ & $290 ; 730$ & $290 ; 730$ & $0.14 ; 3$ & 4 & 0.15 & 0.3 \\
\hline
\end{tabular}

${ }^{a}$ Relative to $\mathrm{CaWO}_{4}$ measured at excitation with $3.2 \mathrm{keV}$ x-ray photons. 
candidates. Due to the high light yield, suitable isotopic abundance and mechanical properties, $\mathrm{CaWO}_{4}$ is currently extensively used as a CPSD in experimental searches for dark matter while $\mathrm{ZnWO}_{4}$ can be implemented readily. This is also relevant for the Ti-doped $\mathrm{Al}_{2} \mathrm{O}_{3}$ scintillator, particular merits of which are its superior phonon properties and a legacy of successful applications as a cryogenic detector. Molybdate crystals also have good potential, though currently their light yield is inferior compared with tungstates. Nonetheless there is no doubt that an essential improvement of these compounds with regard to the light yield is possible.

Looking at the long term perspective, with constantly growing interest in the CPSD technique, one can foresee interest in the practical utilization of the technology in the near future. What seems to be a specialized, or even extreme, requirement today often proves to become a mainstream in a few years' time. This is certainly true for radiation detection technologies. The health sector, especially medical diagnostics, can benefit significantly from the implementation of a new type of detector since there is an urgent need for improvement in energy resolution, which would enhance image quality and reduce doses that patients have to be exposed to. CPSD can offer less than $1 \mathrm{keV}$ resolution in the phonon channel and an unbeatable ability to discriminate between different types of radiation. The latter feature can become decisive considering the strong current interest in neutron imaging for medical purposes. Together with gamma-ray imaging, neutron imaging can enhance diagnostic power significantly. Furthermore this can evolve into promising methods for security inspection, which has the advantage of neutrons interacting predominantly with lighter nuclei, while penetrating materials made of heavier nuclei (e.g. metal shielding). If cryogenic scintillators can be shown to offer a significant advantage over room temperature detectors, they will be implemented as there are developments of automatic cryogenic systems, which will make low temperatures available to a much wider range of users than is possible now. Apparently the search for cryogenic scintillators today is the science-driven initiative that will prepare the grounds for technological progress in respective areas tomorrow.

\section{Acknowledgments}

This work was supported by PPARC, Grant I/S/2001/646 and the Royal Society grant. We are also grateful for support obtained from the SRS Daresbury Laboratory through proposals 41051, 42023 and 45021. The study carried out at HASYLAB was supported by the European CommunityResearch Infrastructure Action under the FP6 'Structuring the European Research Area' Programme through the Integrated Infrastructure Initiative 'Integrating Activity on Synchrotron and Free Electron Laser Science'.

\section{References}

[1] Kraus H 2003 Phil. Trans. R. Soc. Lond. Ser. A 3612581

[2] Fiorini E 2004 Nucl. Instrum. Methods A 5201

[3] Porter F S 2004 Nucl. Instrum. Methods Phys. Res. A 520354

[4] Kraus H 2002 Int. J. Mass Spectrom. 21545
[5] Christ P, Rutzinger S, Koy C, Seidel W, Uchaikin S, Glocker M O and Pröbst F 2004 Eur. J. Mass Spectrom. 10469

[6] Angloher G et al 2005 Astropart. Phys. 23325

[7] Cebrian S et al 2004 Astropart. Phys. 2123

[8] Cozzini C et al 2004 Phys. Rev. C 70064606

[9] de Marcillac P, Coron N, Dambier G, Leblanc J and Moalic J P 2003 Nature $\mathbf{4 2 2} 876$

[10] Alessandrello A et al 1998 Phys. Lett. B 420109

Dafinei I, Diemoz M, Longo E, Peter A and Foldvari I 2005 Nucl. Instrum. Methods Phys. Res. A 554195

[11] Di Stefano P C F et al 2003 J. Appl. Phys. 946887

[12] Coron N, Dambier G, Leblanc E, Leblanc J, de Marcillac P and Moaliac J -P 2004 Nucl. Instrum. Methods A 520159

[13] Bergström L 2000 Rep. Prog. Phys. 63793

[14] Diemand J, Moore B and Stadel J 2005 Nature 433389

[15] Roszkowski L 2000 Nucl. Phys. B Proc. Suppl. 8721

[16] Roszkowski L 2003 Nucl. Phys. B Proc. Suppl. 12430

[17] Meunier P, Bravin M, Bruckmayer B, Giordano S, Loidi M, Meier O, Pröbst F, Seidel W and Sisti M 1999 Appl. Phys. Lett. $\mathbf{7 5} 1335$

[18] Coron N, de Marcillac P, Leblanc J, Dambier G and Moalic J P 2004 Opt. Eng. 431568

[19] Mikhailik V B, Kraus H, Wahl D, Itoh M, Koike M and Bailiff I 2004 Phys. Rev. B 69205110

[20] Mikhailik V B, Bailiff I K, Kraus H, Rodnyi P A and Nincovic J 2004 Radiat. Meas. 38585

[21] Zimmerer G 1991 Nucl. Instrum. Methods Phys. Res. A 308 178

[22] Quinn F, Poolton N, Malins A, Pantos E, Andersen C, Denby P, Dhanak V and Miller G 2003 J. Synchrotron Radiat. 10461

[23] Ninkovic J et al 2005 Nucl. Instrum. Methods Phys. Res. A $\mathbf{5 3 7} 339$

[24] Kraus H, Mikhailik V B and Wahl D 2005 Nucl. Instrum. Methods Phys. Res. A 553522

[25] Kröger F A 1948 Some Aspects of the Luminescence of Solids (Amsterdam: Elsevier)

[26] Botden Th P J 1951 Philips Res. Rep. 6425

[27] Kaminskii A A et al 1999 Appl. Opt. 384533

[28] Ishi M and Kobayashi M 1991 Prog. Cryst. Growth Charact. Mater. 23245

[29] Derenzo S E and Moses W W 1993 Experimental efforts and results in finding new heavy scintillators Heavy Scintillators for Scientific and Industrial Applications ed de Notaristefani F et al (Gif-sur-Yuette, France: Editions Frontières) $\mathrm{p} 125$

[30] Treadaway M J and Powel R C 1974 J. Chem. Phys. 614003

[31] Tyner C E and Drickamer H G 1977 J. Chem. Phys. 674103

[32] Goernik J A, Hakfoort C and Blasse G 1979 Phys. Status Solidi a $\mathbf{5 4} 477$

[33] Blasse G 1980 Struct. Bond. 421

[34] Grasser R, Sharmann A and Strack K-R 1982 J. Lumin. 27263

[35] Reut E G 1985 Izv. Akad. Nauk SSSR Ser. Fizicheskaya 49 2032 (in Russian)

[36] Blasse G and Bokkers G 1983 J. Solid State Chem. 49126

[37] Ovechkin A E, Ryzhikov V D, Tamulatis G and Zukauskas A 1987 Phys. Status Solidi a 103285

[38] Mikhailin V V, Belsky A N, Kamenskikh I A, Kolobanov V N, Orekhanov P A, Shpinkov I N, Spassky D A and Vasil'ev A N 2002 Nucl. Instrum. Methods A 486367

[39] Nagirnyi V, Feldbach E, Jönsson L, Kirm M, Kotlov A, Lushchik A, Nefedov V A and Zadneprovski B I 2002 Nucl. Instrum. Methods A 486395

[40] Spassky D, Ivanov S, Kitaeva I, Kolobanov V, Mikhailin V, Ivleva L and Voronina I 2005 Phys. Status Solidi c 265

[41] Mürk V, Nikl M, Mihokova E and Nitsch K 1997 J. Phys. Condens. Matter 9249

[42] Babin V, Bohacek P, Bender E, Krasnikov A, Mihokova E, Nikl M, Senguttuvan N, Stolovits A, Usuki Y and Zazubovich S 2004 Radiat. Meas. 38533 
[43] Mikhrin S B, Mishin A N, Potapov A S, Rodnyi P A and Voloshinovskii A S 2002 Nucl. Instrum. Methods A 486295

[44] Pankratov V, Millers D, Grigorjeva L, Chernov S and Voloshinovskii A S 2001 J. Lumin. 94-95 427

[45] Mikhailik V B, Kraus H, Miller G, Mykhaylyk M S and Wahl D 2005 J. Appl. Phys. 97083523

[46] Senyshyn A, Kraus H, Mikhailik V B and Yakovyna V 2004 Phys. Rev. B 70214306

[47] Sokolenko E V, Zhukovskii V M, Buyanova E S and Krasnobaev Ya A 1998 Inorg. Mater. 34611

[48] Zhang Y, Holzwarth N A W and Williams R T 1998 Phys. Rev. B 5712738

[49] Abraham Y B, Holzwarth N A W and Williams R T 2000 Phys. Rev. B 621733

[50] Lushchik A, Kirm M and Lushchik C 1995 Radiat. Meas. 24 365

[51] Kraus H, Mikhailik V B, Ramachers Y, Day D, Hutton K B and Telfer J 2005 Phys. Lett. B 61037

[52] Belogurov S et al 2005 IEE Trans. Nucl. Sci. 521131
[53] Rodriguez J A, Hanson J C, Chaturvedi S, Maiti A and Brito J L 2000 J. Chem. Phys. 112935

[54] Bacci M, Porcinai S, Mihokova E, Nikl M and Polak K 2001 Phys. Rev. B 64104302

[55] Itoh M and Sakurai T 2005 Phys. Status Solidi b 242 $\mathrm{R} 52$

[56] Itoh M, Hayakawa K and Oishi S 2001 J. Phys.: Condens. Matter 136853

[57] Mikhailik V B, Kraus H, Wahl D and Mykhaylyk M S 2005 Phys. Status Solidi b 242 R17

[58] Mikhailik V B, Kraus H, Itoh M, Iri D and Uchida M 2005 J. Phys.: Condens. Matter 177209

[59] Bravin M, Bruckmayer M, Bucci C, Cooper S, Giordano S, von Feilitzsch F, Höhne J, Jochum J, Jörgens V and Keeling R 1999 Astropart. Phys. 12107

[60] Mikhailik V B, Kraus H, Mykhaylyk M S and Wahl D 2005 Appl. Phys. Lett. 86101909

[61] Mikhailik V B, Kraus H, Balcerzyk M, Czarnacki W, Moszyñski M, Mykhaylyk M S and Wahl D 2005 Nucl. Instrum. Methods Phys. Res. A 546523 